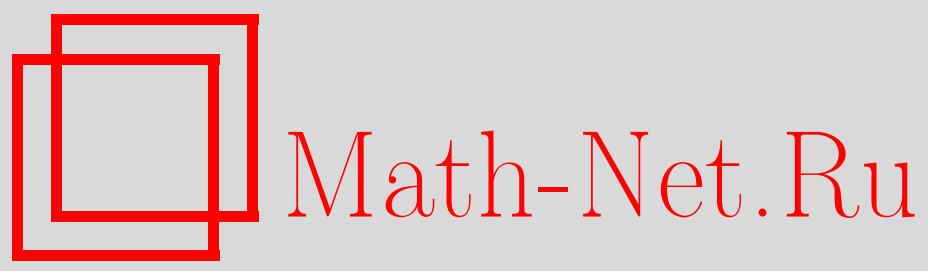

С. В. Асташкин, Д. В. Занин, Е. М. Семёнов, Ф. А. Сукочев, Оператор Круглова и операторы, определяемые случайными перестановками, Функи. анализ и его прил., 2009, том 43, выпуск $2,3-18$

DOI: https://doi.org/10.4213/faa2947

Использование Общероссийского математического портала MathNet.Ru подразумевает, что вы прочитали и согласны с пользовательским соглашением

http://www . mathnet.ru/rus/agreement

Параметры загрузки:

IP : 54.197 .130 .99

26 апреля 2023 г., 13:37:38

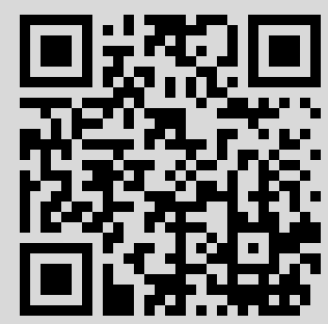


Функционалъный анализ и его приложения

2009, т. 43, вып. 2, с. 3-18

УДК $517.5+517.982$

\section{Оператор Круглова и операторы, определяемые случайными перестановками*}

(c) 2009. С. В. АстАшкин, Д. В. ЗАнин, Е. М. Семенов, Ф. А. Сукочев

Свойство и оператор Круглова играют важную роль при изучении геометрических свойств r. i. пространств. В работе доказано, что ограниченность оператора Круглова в r.i. пространстве эквивалентна равномерной ограниченности в этом пространстве операторов, порожденных случайными перестановками. Показано также, что не существует минимального r. і. пространства со свойством Круглова.

\section{§1. Введение}

Пусть $f$ - случайная величина (измеримая функция), определенная на $[0,1]$. Обозначим через $\pi(f)$ случайную величину на $[0,1]$ с тем же распределением, что и у $\sum_{i=1}^{N} f_{i}$, где $f_{i}-$ независимые копии случайной величины $f$, а $N-$ пуассоновская случайная величина с параметром 1 , независимая от последовательности $\left\{f_{i}\right\}$.

Определение. Говорят, что r. і. пространство $E$ на $[0,1]$ (определение см. в $\S 2)$ обладает свойством Круглова $(E \in \mathbb{K})$, если

$$
f \in E \Longleftrightarrow \pi(f) \in E .
$$

Это свойство было введено и изучалось Браверманом [1], использовавшим при этом некоторые вероятностные конструкции и идеи Круглова [2]. В [3] (см. также [4]) для его изучения был предложен операторный подход.

Пусть $\left\{B_{n}\right\}_{n=1}^{\infty}$ - последовательность измеримых попарно не пересекающихся подмножеств отрезка $[0,1]$ и mes $B_{n}=1 /(e n !)$ (mes - мера Лебега на $\left.[0,1]\right)$. Если $f \in L_{1}[0,1]$, то

$$
K f\left(\omega_{0}, \omega_{1}, \ldots\right)=\sum_{n=1}^{\infty} \sum_{k=1}^{n} f\left(\omega_{k}\right) \chi_{B_{n}}\left(\omega_{0}\right) ;
$$

здесь и всюду далее $\chi_{B}$ - характеристическая функция множества $B$. Тогда $K$ - положительный линейный оператор, $K: L_{1}[0,1] \rightarrow L_{1}(\Omega, P)$, где $(\Omega, P)=$ $\prod_{n=0}^{\infty}([0,1]$, mes). Так как распределения случайных величин $K f$ и $\pi(f)$ одинаковы [3], то $K f$ можно рассматривать как явное представление случайной величины $\pi(f)$. Поэтому, в частности, r. i. пространство $E$ обладает свойством Круглова тогда и только тогда, когда $K$ действует (ограниченным образом) из $E$ в $E(\Omega, P)[3]$.

* Первый автор частично поддержан грантом РФФИ №07-01-96603; третий автор частично поддержан грантом РФФИ №08-01-00226-а; четвертый автор частично поддержан Австралийским научным советом. 
Через $K f$ мы также будем обозначать другую случайную величину, определенную на $[0,1]$ и имеющую то же распределение, что и величина, введенная формулой (1) (см. [3]). Обозначим через $f^{*}$ перестановку функции $|f|$ в убывающем порядке, т. е. $f^{*}(t)$ не возрастает на $[0,1]$ и равноизмерима с $|f(t)|$. Если $f \in L_{1}[0,1],\left\{B_{n}\right\}$ - указанная выше последовательность подмножеств отрезка $[0,1]$ и $f_{n, 1}, \ldots, f_{n, n}, \chi_{B_{n}}-$ набор независимых функций для каждого $n \in \mathbb{N}$, такой, что $f_{n, k}^{*}=f^{*}$ для всех $n \in \mathbb{N}$ и $k=1, \ldots, n$, то $K f(t)$ определяется как некоторая перестановка функции

$$
\sum_{n=1}^{\infty} \sum_{k=1}^{n} f_{n, k}(t) \chi_{B_{n}}(t) \quad(0 \leqslant t \leqslant 1) .
$$

Подчеркнем, что $K$ - это линейный оператор, действующий из пространства $L_{1}[0,1]$ в пространство $L_{1}(\Omega, P)$. Когда мы говорим, что $K$ ограничен в r. i. пространстве $X$ на $[0,1]$, то эта ограниченность понимается как ограниченность линейного отображения $K$ из пространства $X[0,1]$ в пространство $X(\Omega, P)$. Из определений оператора $K$ и r. i. пространства сразу следует (см. также $[1,1.6$, c. 11]), что $\|K f\|_{E} \geqslant e^{-1}\|f\|_{E}(f \in E)$ для любого r. і. пространства $E$. В [3] было показано, что оператор $K$ играет важную роль при получении оценок норм сумм независимых функций через нормы сумм их дизъюнктных копий (эти оценки имеют многочисленные применения). На этом пути в [3] было найдено усиление известных результатов Джонсона и Шехтмана [5].

Хорошо известно (см., например, [2] или [1]), что пространство Орлича $\exp L_{1}$, построенное по функции $e^{t}-1$, обладает свойством Круглова. Отсюда следует, что то же самое верно и для его сепарабельной части $\left(\exp L_{1}\right)_{0}$ (определения см. ниже). Действительно, так как $K$ ограничен в $\exp L_{1}$, то $K\left(\left(\exp L_{1}\right)_{0}\right) \subset \overline{K\left(L_{\infty}\right)}$ (замыкание берется относительно нормы в $\left.\exp L_{1}\right)$. Одновременно $K\left(L_{\infty}\right) \subset$ $\left(\exp L_{1}\right)_{0} \quad[3$, теорема 4.4]. Следовательно, ввиду замкнутости множества $\left(\exp L_{1}\right)_{0}$ в $\exp L_{1}$ оператор $K$ ограничен в $\left(\exp L_{1}\right)_{0}$. Заметим, что для всех известных до сих пор r.i. пространств $E$ со свойством Круглова имело место вложение $E \supset\left(\exp L_{1}\right)_{0}$. Это, а также некоторые результаты работы [3] (см. теорему 7.2) делали весьма правдоподобной гипотезу о том, что $\left(\exp L_{1}\right)_{0}$ минимальное среди r.i. пространств со свойством Круглова. Тем не менее в первой части работы мы покажем, что это неверно. Более того, будет доказано, что для всякого r.i. пространства $E \in \mathbb{K}$ существует пространство Марцинкевича $M_{\psi} \varsubsetneqq X$, также обладающее свойством Круглова (следствие 3 ). В определенном смысле совершенно иначе обстоит дело с обладающими свойством Круглова пространствами Лоренца: любое такое пространство содержит ехр $L_{1}$ (теорема 4).

Квапень и Шютт в работе [6] исследовали свойства случайных перестановок, применив их затем к изучению геометрии банаховых пространств. Операторный подход к этим вопросам был развит в работах [7] и [8] и позволил обобщить результаты Квапеня и Шютта. Там было введено следующее семейство операторов. Пусть $n \in \mathbb{N}$ и $S_{n}$ - множество перестановок чисел $1, \ldots, n$. Отождествим $S_{n}$ с множеством $\{1, \ldots, n !\}$ (произвольным образом) и определим сначала оператор $A_{n}$, действующий из $\mathbb{R}^{n}$ в $\mathbb{R}^{n !}$ : если $x=\left(x_{1}, \ldots, x_{n}\right) \in \mathbb{R}^{n}$ и $\pi$ - произвольная перестановка из $S_{n}$, то компонента вектора $A_{n} x$, соответствующая 
перестановке $\pi$ (отождествляемой с элементом множества $\{1, \ldots, n !\})$, определяется по формуле

$$
\left(A_{n} x\right)_{\pi}:=\sum_{i: \pi(i)=i} x_{i} .
$$

Для функции $x(t) \in L_{1}[0,1]$ определим вектор $B_{n} x$ длины $n$ с координатами $\left(B_{n} x\right)_{i}=n \int_{(i-1) / n}^{i / n} x(t) d t, i=1, \ldots, n$. Оператор $B_{n}$ имеет правый обратный $C_{n}$ $\left(B_{n} C_{n} x=x\right.$ для $\left.x \in \mathbb{R}^{n}\right)$, сопоставляющий каждому вектору длины $n$ функцию, постоянную на отрезках $[(i-1) / n, i / n]$. Наконец, определим оператор

$$
T_{n}=C_{n !} A_{n} B_{n}
$$

Ясно, что $T_{n}$ для каждого $n \in \mathbb{N}$ - линейный положительный оператор, действующий из $L_{1}[0,1]$ в пространство ступенчатых функций. Нетрудно показать также, что

$$
\left\|T_{n} x\right\|_{L_{1}}=\|x\|_{L_{1}}
$$

для всех $x \in L_{1}[0,1], x \geqslant 0$. Через $T_{n}$ мы будем также иногда обозначать оператор $C_{n !} A_{n}$, определенный аналогичным образом на $\mathbb{R}^{n}$ (это не приведет к недоразумению). Если $x=\left(x_{1}, \ldots, x_{n}\right) \in \mathbb{R}^{n}$ и $E$ - произвольное r. i. пространство на $[0,1]$, то $\|x\|_{E}$ понимается как

$$
\left\|C_{n} x\right\|_{E}=\left\|\sum_{k=1}^{n} x_{k} \chi_{\left(\frac{k-1}{n}, \frac{k}{n}\right)}\right\|_{E} .
$$

В работе [8] было доказано, что равномерная ограниченность операторов, порожденных случайными перестановками, на множестве квадратных матриц сводится к аналогичному свойству операторов $T_{n}$. Одновременно обнаружилось интересное обстоятельство: несмотря на внешнее отсутствие какой-либо связи между операторами $K$ и $T_{n}$, критерии ограниченности оператора $K$ и равномерной ограниченности операторов $T_{n}(n \in \mathbb{N})$ в пространстве Лоренца $\Lambda_{\varphi}$, найденные в [3] и [8] соответственно, оказались одинаковыми. А именно, оба они состоят в выполнении одного и того же условия:

$$
M:=\sup _{0<t \leqslant 1} \frac{1}{\varphi(t)} \sum_{k=1}^{\infty} \varphi\left(\frac{t^{k}}{k !}\right)<\infty .
$$

В связи с этим возникла гипотеза о том, что ограниченность оператора $K$ в произвольном r. i. пространстве $E$ также эквивалентна условию $\sup _{n}\left\|T_{n}\right\|_{E}<\infty$. Вторая часть работы посвящена доказательству этого утверждения. Оно основано на комбинаторных рассуждениях и связано с получением оценок для соответствующих функций распределения. Доказанный результат приводит к ряду полезных следствий как для оператора $K$, так и для операторов $T_{n}$. В частности, следствие 13 показывает (уточняя теорему 19 из [8]), что равномерная ограниченность операторов $T_{n}$ в экспоненциальных пространствах Орлича $\exp L_{p}$ эквивалентна тому, что $p \leqslant 1$.

Авторы выражают благодарность рецензенту, предложения и замечания которого позволили улучшить окончательный текст статьи, в частности, упростить определение операторов $T_{n}$ и доказательство леммы 7. 


\section{§2. Определения и обозначения}

Банахово пространство $E$ измеримых на $[0,1]$ функций называется перестановочно-инвариантным, или симметричным, или r. i. пространством, если

1) из $|x(t)| \leqslant|y(t)|$ для $t \in[0,1]$ и $y \in E$ следует, что $x \in E$ и $\|x\|_{E} \leqslant\|y\|_{E}$;

2 ) из равноизмеримости функции $x$ и функции $y \in E$, т. е. равенства

$$
\operatorname{mes}\{t \in[0,1]:|x(t)|>\tau\}=\operatorname{mes}\{t \in[0,1]:|y(t)|>\tau\} \quad(\tau>0),
$$

следует, что $x \in E$ и $\|x\|_{E}=\|y\|_{E}$.

Для любого r.i. пространства $E$ имеют место непрерывные вложения $L_{\infty} \subset$ $E \subset L_{1}$ и, если $\left\|\chi_{(0,1)}\right\|_{E}=1$, то $\|x\|_{L_{1}} \leqslant\|x\|_{E} \leqslant\|x\|_{L_{\infty}}\left(x \in L_{\infty}\right)$. Для каждого $\tau>0$ оператор растяжения $\sigma_{\tau} x(t):=x(t / \tau) \cdot \chi_{[0, \min (1, \tau)]}(t)(0 \leqslant t \leqslant 1)$ ограничен в $E$ и $\left\|\sigma_{\tau}\right\|_{E} \leqslant \max (1, \tau)$.

Пространство $E^{\prime}$ измеримых функций $x$ на $[0,1]$, для которых

$$
\|x\|_{E^{\prime}}=\sup _{\|y\|_{E} \leqslant 1} \int_{0}^{1} x(t) y(t) d t<\infty,
$$

называется двойственным к $E$ пространством; оно также является r.i. пространством. Следуя [9, 2.а.1], мы будем предполагать, что $E$ сепарабельно или совпадает со своим вторым двойственным пространством $E^{\prime \prime}$. Тогда $E^{\prime \prime}$ в обоих случаях содержит $E$ как замкнутое подпространство и вложение $E \subset E^{\prime \prime}$ изометрично. Если $E$ сепарабельно, то $E^{\prime}$ совпадает с сопряженным пространством $E^{*}$. Через $E_{0}$ будет обозначаться сепарабельная часть r. i. пространства $E$, т. е. замыкание в $E$ пространства $L_{\infty}$. Пространство $E_{0}$ совпадает с $L_{\infty}$, если и только если $E=L_{\infty}$. В случае когда $E \neq L_{\infty}, E_{0}$ является сепарабельным r. i. пространством.

Напомним, что слабая сходимость распределений измеримых на $[0,1]$ функций $x_{n}(t)$ к распределению функции $x(t)\left(x_{n} \Rightarrow x\right)$ означает, что для любой непрерывной и ограниченной на $(0, \infty)$ функции $y(t)$

$$
\lim _{n \rightarrow \infty} \int_{-\infty}^{\infty} y(t) d \operatorname{mes}\left\{s: x_{n}(s)<t\right\}=\int_{-\infty}^{\infty} y(t) d \operatorname{mes}\{s: x(s)<t\} .
$$

Если $E$ есть r.і. пространство, $x_{n} \in E(n \in \mathbb{N}), \lim _{\sup _{n \rightarrow \infty}}\left\|x_{n}\right\|_{E}=C<\infty$ и $x_{n} \Rightarrow x$, то $x \in E^{\prime \prime}$ и $\|x\|_{E^{\prime \prime}} \leqslant C$ [1, предложение 1.5$]$.

На множестве суммируемых функций вводится отношение полуупорядоченности $\prec$, играющее важную роль в теории r. i. пространств. Мы будем писать $x \prec y$, если

$$
\int_{0}^{\tau} x^{*}(t) d t \leqslant \int_{0}^{\tau} y^{*}(t) d t
$$

для всех $\tau \in[0,1]$. Здесь и далее $x^{*}(t)$ - невозрастающая непрерывная слева перестановка функции $|x(t)|$, т. е.

$$
x^{*}(t)=\inf \{\tau \geqslant 0: \operatorname{mes}\{s \in[0,1]:|x(s)|>\tau\}<t\} \quad(0<t \leqslant 1) .
$$

Если $x \prec y$ и $y$ принадлежит некоторому r.i. пространству $E$, то $x \in E$ и $\|x\|_{E} \leqslant\|y\|_{E}$. 
Приведем наиболее важные примеры r. i. пространств. Если $M(u)-$ возрастающая выпуклая на $[0, \infty)$ функция, $M(0)=0$, то через $L_{M}$ будет обозначаться пространство Орлича с нормой

$$
\|x\|_{L_{M}}=\inf \left\{\lambda>0: \int_{0}^{1} M\left(\frac{|x(t)|}{\lambda}\right) d t \leqslant 1\right\} .
$$

Функция $M_{p}(u)=e^{u^{p}}-1$ выпукла при $p \geqslant 1$ и эквивалентна некоторой выпуклой функции при $0<p<1$. Пространство $L_{M_{p}}$ будет обозначаться через $\exp L_{p}$.

Если $\varphi(t)$ - возрастающая вогнутая функция на $[0,1], \varphi(0)=0$, то $\Lambda_{\varphi}-$ пространство Лоренца с нормой

$$
\|x\|_{\Lambda_{\varphi}}=\int_{0}^{1} x^{*}(t) d \varphi(t)
$$

а $M_{\varphi}$ - пространство Марцинкевича с нормой

$$
\|x\|_{M_{\varphi}}=\sup _{0<t \leqslant 1} \frac{1}{\varphi(t)} \int_{0}^{t} x^{*}(s) d s .
$$

Все изложенные выше сведения о r. i. пространствах содержатся в монографиях [9], [10].

Всюду далее supp $f$ - носитель функции $f$, т. е. множество $\{t: f(t) \neq 0\}$. Кроме того, мы пишем $F \asymp G$, если $C^{-1} F \leqslant G \leqslant C F$, где константа $C>0$ не зависит от аргументов величин $F$ и $G$. И наконец, через $|A|$ будет обозначаться количество элементов конечного множества $A$.

\section{§3. Пространства Марцинкевича и Лоренца «вблизи» $\exp L_{1}$}

Теорема 1. Существует такое семейство пространств Мариинкевича $\left\{M_{\psi_{\varepsilon}}\right\}_{0<\varepsilon<1}$, что $M_{\psi_{\varepsilon}} \subset M_{\psi_{\delta}}$ для всех $0<\varepsilon \leqslant \delta<1$ и что

1) $M_{\psi_{\varepsilon}} \in \mathbb{K}, 0<\varepsilon<1$;

2) $M_{\psi_{\varepsilon}} \subset E$ для каждого r.i. пространства $E \in \mathbb{K}$ и всех достаточно ма$\operatorname{\imath ъx} \varepsilon$;

3) функции $\psi_{\varepsilon}$ попарно не эквивалентны, точнее,

$$
\lim _{t \rightarrow 0} \frac{\psi_{\varepsilon}(t)}{\psi_{\delta}(t)}=0, \quad \text { если } 0<\varepsilon<\delta<1 ;
$$

4) $M_{\psi_{\varepsilon}} \varsubsetneqq\left(\exp L_{1}\right)_{0}$ для достаточно мальх $\varepsilon>0$.

Для доказательства нам потребуется следующее простое утверждение.

Лемма 2. Для произвольной функиии $f \in L_{1}[0,1]$

$$
\lim _{n \rightarrow \infty} \operatorname{mes}\left(\operatorname{supp} K^{n} f\right)=0 \text {. }
$$

Доказательство. Так как оператор $K$ положителен, можно считать, что $f \geqslant 0$ и что $\operatorname{mes}(\operatorname{supp} f)=1$. Тогда если $a_{n}:=\operatorname{mes}\left\{t: K^{n} f(t)=0\right\}(n \in \mathbb{N})$, то по определению оператора $K$ (см. соотношение $(2)) a_{1}=1 / e$ и

$$
a_{n+1}=\frac{1}{e}+\frac{1}{e} \sum_{k=1}^{\infty} \frac{a_{n}^{k}}{k !}=e^{a_{n}-1} \quad(n=1,2, \ldots) .
$$


Легко видеть, что последовательность $\left\{a_{n}\right\}$ возрастает и $a_{n} \in(0,1)$. Так как $f(x):=e^{x-1}-x$ убывает на $[0,1]$, то функция $e^{x-1}$ имеет на этом отрезке единственную неподвижную точку $x=1$. Следовательно, $\lim _{n \rightarrow \infty} a_{n}=1$, и утверждение доказано.

Доказательство теоремы 1. Рассмотрим функции $h_{n}=\left(K^{n} 1\right)^{*}, n \geqslant 0$. Так как оператор $K$ переводит равноизмеримые функции в равноизмеримые, то

$$
\left(K h_{n}\right)^{*}=h_{n+1} .
$$

Далее, по лемме $2 \operatorname{mes}\left(\operatorname{supp} h_{n}\right) \rightarrow 0$ при $n \rightarrow \infty$ и, значит, ряд

$$
g_{\varepsilon}=\sum_{n=0}^{\infty} \varepsilon^{n} h_{n}
$$

сходится всюду на $(0,1]$ для каждого $\varepsilon>0$, а функция $g_{\varepsilon}$ убывает. Кроме того, из определения оператора $K$ (см. (2)) следует, что $\|K\|_{L_{1}}=1$. Поэтому если $0<\varepsilon<1$, то ряд (8) сходится в $L_{1}$ и $g_{\varepsilon} \in L_{1}$. Покажем теперь, что утверждения теоремы справедливы для семейства $\left\{M_{\psi_{\varepsilon}}\right\}_{\varepsilon>0}$, где $\psi_{\varepsilon}(t)=\int_{0}^{t} g_{\varepsilon}(s) d s(0 \leqslant$ $t \leqslant 1)$.

1. Докажем, что оператор $K$ ограничен в $M_{\psi_{\varepsilon}}$. Так как крайние точки единичного шара в этом пространстве равноизмеримы с функцией $g_{\varepsilon}$ [11], то для этого достаточно показать, что $K g_{\varepsilon} \in M_{\psi_{\varepsilon}}$. Ввиду ограниченности оператора $K$ в $L_{1}$

$$
K g_{\varepsilon}=\sum_{n=0}^{\infty} \varepsilon^{n} K h_{n} \prec \sum_{n=0}^{\infty} \varepsilon^{n} h_{n+1} \leqslant \frac{1}{\varepsilon} \sum_{n=0}^{\infty} \varepsilon^{n} h_{n}=\frac{1}{\varepsilon} g_{\varepsilon},
$$

где первое неравенство вытекает из (7) и хорошо известного свойства полуупорядоченности Харди-Литтлвуда (см., например, [10, §2.2]). Отсюда следует, что $K g_{\varepsilon} \in M_{\psi_{\varepsilon}}$.

2. Предположим теперь, что $E \in \mathbb{K}$. Тогда, как уже отмечалось, $C=\|K\|_{E \rightarrow E}$ $<\infty$. Очевидно, что $\left\|h_{n}\right\|_{E} \leqslant C^{n}\|1\|_{E}$. Следовательно, для всех $\varepsilon<C^{-1}$ ряд (8) сходится в $E$ и $g_{\varepsilon} \in E$. Кроме того, так как пространство $E$ сепарабельно или $E=E^{\prime \prime}$, то из $x \in E$ и $y \prec x$ следует, что $y \in E$ и $\|y\|_{E} \leqslant\|x\|_{E}$. Таким образом, вместе с $g_{\varepsilon}$ весь единичный шар пространства Марцинкевича $M_{\psi_{\varepsilon}}$ содержится в $E$, откуда вытекает, что $M_{\psi_{\varepsilon}} \subset E$.

3. Пусть, как и ранее, функция $g_{\varepsilon}$ определяется соотношением $(8)$ и $0<\varepsilon<\delta$. Так как в силу доказательства теоремы 7.2 в [3]

$$
\lim _{t \rightarrow 0} \frac{h_{n+1}(t)}{h_{n}(t)}=\infty
$$

то для любого $m=1,2, \ldots$

$$
\begin{aligned}
\limsup _{t \rightarrow 0} \frac{g_{\varepsilon}(t)}{g_{\delta}(t)} & =\limsup _{t \rightarrow 0}\left(\sum_{n=1}^{\infty} \varepsilon^{n} h_{n}(t)\right) \cdot\left(\sum_{n=1}^{\infty} \delta^{n} h_{n}(t)\right)^{-1}=\cdots \\
& =\limsup _{t \rightarrow 0}\left(\sum_{n=m}^{\infty} \varepsilon^{n} h_{n}(t)\right) \cdot\left(\sum_{n=m}^{\infty} \delta^{n} h_{n}(t)\right)^{-1} \leqslant\left(\frac{\varepsilon}{\delta}\right)^{m} .
\end{aligned}
$$

Таким образом, $\lim _{t \rightarrow 0} g_{\varepsilon}(t) / g_{\delta}(t)=0$, откуда следует (6). 
4. Как уже говорилось во введении, $K$ ограниченно действует в пространстве $\left(\exp L_{1}\right)_{0}$. Поэтому достаточно применить уже доказанные второе и третье утверждения.

Пусть $\varphi_{n}(t)=\int_{0}^{t} h_{n}(s) d s(0 \leqslant t \leqslant 1)$ и $M_{\varphi_{n}}-$ пространство Марцинкевича, порожденное этой функцией. Тогда $M_{\varphi_{n}} \subset M_{\varphi_{n+1}} \subset\left(\exp L_{1}\right)_{0}(n=1,2, \ldots)$, и в определенном смысле пространства $M_{\varphi_{n}}$ можно считать «приближениями» пространства $\left(\exp L_{1}\right)_{0}$. В работе [3] (см. теорему 7.2) доказано, что для любого r. i. пространства $E \in \mathbb{K}$ и всех $n=1,2, \ldots$ имеет место вложение $M_{\varphi_{n}} \subset E$. В связи с этим возникла гипотеза о том, что $\left(\exp L_{1}\right)_{0}-$ наименьшее среди r. i. пространств со свойством Круглова. Тем не менее следующее утверждение, вытекающее из только что доказанной теоремы, показывает, что эта гипотеза не верна.

Следствие 3. Для любого r. $i$. пространства $E \in \mathbb{K}$ существует такое r. $i$. пространство $F \in \mathbb{K}$, что $F \varsubsetneqq E$.

Покажем, что в отличие от пространств Марцинкевича все пространства Лоренца со свойством Круглова лежат «по одну сторону» от $\exp L_{1}$.

Теорема 4. Пусть $\varphi-$ возрастающая вогнутая на $[0,1]$ функиия, $\varphi(0)=0$. Если $\Lambda_{\varphi} \in \mathbb{K}, \operatorname{mo~} \Lambda_{\varphi} \supset \exp L_{1}$.

Здесь мы также начнем с леммы.

Лемма 5. Если возрастающая вогнутая на $[0,1]$ функиия $\varphi, \varphi(0)=0$, удовлетворяет условию (5), то

$$
\sum_{k=1}^{\infty} \varphi\left(2^{-k}\right) \leqslant A \varphi(1)
$$

где $A>0$ зависит лишь от константы $M$ из (5).

Доказательство. Ввиду (5) для всех $i \in \mathbb{N}$

$$
\sum_{j=1}^{\infty} \varphi\left(2^{-i j} j^{-j}\right) \leqslant M \varphi\left(2^{-i}\right)
$$

или

$$
\sum_{j=1}^{\infty} \varphi\left(2^{-j\left(i+\left[\log _{2} j\right]\right)}\right) \leqslant M \varphi\left(2^{-i}\right)
$$

Прямые оценки показывают, что величина

$$
\alpha_{n}:=\left|\left\{(i, j) \in \mathbb{N}^{2}: j\left(i+\left[\log _{2} j\right]\right) \leqslant n\right\}\right|
$$

удовлетворяет соотношению $\lim _{n \rightarrow \infty} \alpha_{n} / n=0$. Поэтому $\alpha_{n} \geqslant(M+1) n$ для некоторого $m \in \mathbb{N}$ и всех $n \geqslant m$. Так как функция $\varphi$ возрастает, то отсюда и из (10) для произвольного $l>m$ следует

$$
(M+1) \sum_{n=m}^{l} \varphi\left(2^{-n}\right) \leqslant \sum_{i=1}^{l} \sum_{j=1}^{\infty} \varphi\left(2^{-j\left(i+\left[\log _{2} j\right]\right)}\right) \leqslant M \sum_{i=1}^{l} \varphi\left(2^{-i}\right),
$$

откуда

$$
\sum_{n=m}^{l} \varphi\left(2^{-n}\right) \leqslant M \sum_{i=1}^{m-1} \varphi\left(2^{-i}\right)
$$


Неравенство (9) является теперь непосредственным следствием того, что $l>m$ произвольно, а $m$ не зависит от $\varphi$.

Доказательство теоремы 4. Как уже отмечалось во введении, условие $\Lambda_{\varphi} \in \mathbb{K}$ эквивалентно соотношению (5) [3]. Поэтому по только что доказанной лемме выполнено неравенство (9). Кроме того, ввиду [12]

$$
\|x\|_{\exp L_{1}} \asymp \sup _{0<t \leqslant 1} x^{*}(t) \log _{2}^{-1}(2 / t),
$$

и, значит, для доказательства вложения $\Lambda_{\varphi} \supset \exp L_{1}$ достаточно показать, что $\log _{2}(2 / t) \in \Lambda_{\varphi}$. Но последнее вытекает из оценок

$$
\begin{aligned}
\left\|\log _{2}(2 / t)\right\|_{\Lambda_{\varphi}} & =\int_{0}^{1} \log _{2}(2 / t) d \varphi(t)=\sum_{k=1}^{\infty} \int_{2^{-k}}^{2^{-k+1}} \log _{2}(2 / t) d \varphi(t) \\
& \leqslant \sum_{k=1}^{\infty}(k+1)\left(\varphi\left(2^{-k+1}\right)-\varphi\left(2^{-k}\right)\right)=2 \varphi(1)+\sum_{k=1}^{\infty} \varphi\left(2^{-k}\right)<\infty .
\end{aligned}
$$

\section{§4. Оценки функций распределения}

Далее для нас будет полезна следующая аппроксимация функции $K f$, где $f$ - произвольная измеримая функция на $[0,1]$.

Пусть $m \in \mathbb{N}, g_{m}(t)=\sigma_{1 / m} f(t)$ и $\left\{h_{m, i}\right\}_{i=1}^{m}-$ набор независимых функций, равноизмеримых с функцией $g_{m}$. Тогда последовательность

$$
H_{m} f(t)=\sum_{i=1}^{m} h_{m, i}(t) \quad(0 \leqslant t \leqslant 1)
$$

при $m \rightarrow \infty$ слабо сходится (в смысле сходимости распределений) к $K f$ (см. [1, 1.6 , с. 11]) или [3, теорема 3.5$]$.

В частности, если $n \in \mathbb{N}, a_{k} \geqslant 0(1 \leqslant k \leqslant n)$ и

$$
f_{a}(t)=\sum_{k=1}^{n} a_{k} \chi_{\left(\frac{k-1}{n}, \frac{k}{n}\right)}(t) \quad(0 \leqslant t \leqslant 1),
$$

TO

$$
g_{m}(t)=\sigma_{\frac{1}{m}} f_{a}(t)=\sum_{k=1}^{n} a_{k} \chi_{\left(\frac{k-1}{n m}, \frac{k}{n m}\right)}(t) \quad(m \in \mathbb{N}) .
$$

В этом случае мы положим

$$
H_{m} a(t):=H_{m} f_{a}(t)=\sum_{i=1}^{m} h_{m, i}(t) .
$$

Кроме того, через $\mathrm{Ch}(r)$ будет обозначаться число перестановок $\pi$ множества $\{1, \ldots, r\}$, таких, что $\pi(i) \neq i$ для всех $i=1, \ldots, r$. Как известно (см., например, $[13$, c. 20]),

$$
\frac{1}{3} r ! \leqslant \operatorname{Ch}(r) \leqslant r ! \quad(r \in \mathbb{N}) .
$$


Сравним сначала функции $H_{m} a$ и $T_{n m} b$, где

$$
b=(\underbrace{a_{1}, \ldots, a_{1}}_{m}, \underbrace{a_{2}, \ldots, a_{2}}_{m}, \ldots, \underbrace{a_{n}, \ldots, a_{n}}_{m}) .
$$

Лемма 6. Для любъх $n, m \in \mathbb{N} u \tau>0$

$$
\operatorname{mes}\left\{t: H_{m} a(t)>\tau\right\} \leqslant 3 \operatorname{mes}\left\{t: T_{n m} b(t)>\tau\right\} .
$$

Доказательство. Функция $H_{m} a(t)$ (соответственно $T_{n m} b(t)$ ) принимает лишь значения вида $\sum_{i=1}^{n} k_{i} a_{i}$, где $k_{i} \in \mathbb{Z}, k_{i} \geqslant 0$ для всех $i=1, \ldots, n$ и $\sum_{i=1}^{n} k_{i} \leqslant m$ (соответственно $\left.\sum_{i=1}^{n} k_{i} \leqslant m n\right)$. Поэтому для доказательства леммы достаточно показать, что

$$
\operatorname{mes}\left\{t: H_{m} a(t)=\sum_{i=1}^{n} k_{i} a_{i}\right\} \leqslant 3 \operatorname{mes}\left\{t: T_{n m} b(t)=\sum_{i=1}^{n} k_{i} a_{i}\right\}
$$

для любого набора $k_{i} \in \mathbb{N}, \sum_{i=1}^{n} k_{i}=q \leqslant m$. Заметим также, что достаточно рассмотреть случай, когда

$$
\sum_{i=1}^{n} k_{i} a_{i} \neq \sum_{i=1}^{n} k_{i}^{\prime} a_{i} \text { при условии, что }\left(k_{1}, \ldots, k_{n}\right) \neq\left(k_{1}^{\prime}, \ldots, k_{n}^{\prime}\right) \text {. }
$$

Поэтому $H_{m} a(t)$ равна сумме $\sum_{i=1}^{n} k_{i} a_{i}$ лишь тогда, когда ровно $k_{i}$ (соответственно $m-q)$ из функций $h_{m, j}(t)(j=1, \ldots, m)$ обращаются в $a_{i}$ (соответственно в 0$)$. Так как $h_{m, j}$ попарно независимы, то

$$
\begin{aligned}
\operatorname{mes}\left\{t: H_{m} a(t)=\sum_{i=1}^{n} k_{i} a_{i}\right\} & =C_{m}^{m-q, k_{1}, \ldots, k_{n}}\left(1-\frac{1}{m}\right)^{m-q}\left(\frac{1}{m n}\right)^{k_{1}+\cdots+k_{n}} \\
& \leqslant C_{m}^{m-q, k_{1}, \ldots, k_{n}}\left(\frac{1}{m n}\right)^{q}
\end{aligned}
$$

где

$$
C_{m}^{m-q, k_{1}, \ldots, k_{n}}=\frac{m !}{(m-q) ! k_{1} ! \cdots k_{n} !} .
$$

С другой стороны, ввиду (3) и (14)

$$
\begin{aligned}
\operatorname{mes}\left\{t: T_{m n} b(t)=\sum_{i=1}^{n} k_{i} a_{i}\right\} & =C_{m}^{k_{1}} \cdots C_{m}^{k_{n}} \operatorname{Ch}(m n-q) \frac{1}{(m n) !} \\
& \geqslant \frac{(m !)^{n}(m n-q) !}{3\left(m-k_{1}\right) ! \cdots\left(m-k_{n}\right) ! k_{1} ! \cdots k_{n} !(m n) !} .
\end{aligned}
$$

Так как $\left(m-k_{1}\right) ! \cdots\left(m-k_{n}\right) ! \leqslant(m !)^{n-1}(m-q) !$ и $(m n-q) ! /(m n) ! \geqslant 1 /(m n)^{q}$, TO

$$
\begin{aligned}
\operatorname{mes}\left\{t: T_{m n} b(t)=\sum_{i=1}^{n} k_{i} a_{i}\right\} & \geqslant \frac{m !(m n-q) !}{3 k_{1} ! \cdots k_{n} !(m-q) !(m n) !} \\
& \geqslant \frac{m !}{3(m-q) ! k_{1} ! \cdots k_{n} !} \cdot \frac{1}{(m n)^{q}} .
\end{aligned}
$$

Отсюда и из неравенства (15) следует утверждение леммы. 
Лемма 7. Если $n, k \in \mathbb{N}, n \geqslant 4, k \leqslant n, m o$

$$
\frac{(n-k) !}{n !} \leqslant 2 \frac{(k-1) !}{n^{k}} .
$$

Доказательство. Так как $j(n-j)>n$ при $2 \leqslant j \leqslant n-2$, то

$$
\frac{n^{k}(n-k) !}{n !(k-1) !}=\prod_{j=1}^{k-1} \frac{n}{j(n-j)} \leqslant\left(\frac{n}{n-1}\right)^{2}<2 .
$$

Продолжим начатое в лемме 6 изучение взаимосвязей между функциями распределения случайных величин $T_{n} a$ и $H_{m} a$ (см. соотношения (3) и (13)). В отличие от оценки, доказанной в лемме 6 для любых $m, n$, противоположное неравенство можно получить только асимптотически при $m \rightarrow \infty$.

Лемма 8. Пусть $n \in \mathbb{N}, a=\left(a_{1}, \ldots, a_{n}\right) \geqslant 0 u \tau>0$. Тогда для достаточно больиих $m \in \mathbb{N}$

$$
\operatorname{mes}\left\{t: T_{n} a(t)>\tau\right\} \leqslant 12 \operatorname{mes}\left\{t: 2 H_{m} a(t)>\tau\right\} .
$$

Доказательство. Предполагая сначала, что $n \geqslant 4$, введем следующие обозначения: $A=\{1, \ldots, n\}$ и $S(U):=\sum_{j \in U} a_{j}$ для произвольного $U \subset A$. Без ограничения общности можно считать, что $n=2 s(s \in \mathbb{N}), a_{i}>0$ и $S\left(U_{1}\right) \neq$ $S\left(U_{2}\right)$, если $U_{1} \neq U_{2}$. Кроме того, пусть $\mathscr{A}_{i}$ - семейство всех $U \subset A$, таких, что $|U|=i(i=1, \ldots, n)$. Тогда $\mathscr{A}=\bigcup_{i=1}^{n} \mathscr{A}_{i}$ состоит из всех непустых подмножеств множества $A$. Представим семейство $\mathscr{A}$ несколько иначе.

Пусть $U \in \mathscr{A}_{k}$ для некоторого $k=1, \ldots, s$. Рассмотрим семейство $\mathscr{A}_{U}$ (соответственно $\left.\mathscr{B}_{U}\right)$, состоящее из всех множеств $V \subset A$, таких, что $V \supset U, V \in \mathscr{A}_{2 k}$ (соответственно $\left.V \in \mathscr{A}_{2 k-1}\right)$ и $S(V \backslash U) \leqslant S(U)$. Так как

$$
\bigcup_{U \in \mathscr{A}_{k}} \mathscr{A}_{U}=\mathscr{A}_{2 k} \quad \text { и } \bigcup_{U \in \mathscr{A}_{k}} \mathscr{B}_{U}=\mathscr{A}_{2 k-1} \quad(k=1, \ldots, s),
$$

то

$$
\mathscr{A}=\bigcup_{k=1}^{s} \bigcup_{U \in \mathscr{A}_{k}}\left(\mathscr{A}_{U} \cup \mathscr{B}_{U}\right) .
$$

Из определения семейств $\mathscr{A}_{U}$ и $\mathscr{B}_{U}$ следует также, что для $V \in \mathscr{A}_{U} \cup \mathscr{B}_{U}$

$$
S(U) \leqslant S(V) \leqslant 2 S(U) .
$$

Заметим, что $T_{n} a(t)$ - ступенчатая функция со значениями $S(V)$, где $V \in \mathscr{A}$. При этом, если $|V|=r$, то ввиду (14)

$$
\operatorname{mes}\left\{t: T_{n} a(t)=S(V)\right\}=\frac{\operatorname{Ch}(n-r)}{n !} \leqslant \frac{(n-r) !}{n !} .
$$

Кроме того, если $|U|=k(k=1, \ldots, s)$, то

$$
\left|\mathscr{A}_{U}\right| \leqslant C_{n-k}^{k}=\frac{(n-k) !}{k !(n-2 k) !}
$$

и, аналогично,

$$
\left|\mathscr{B}_{U}\right| \leqslant C_{n-k}^{k-1}=\frac{(n-k) !}{(k-1) !(n-2 k+1) !} .
$$


Поэтому из (16) и (17) получаем

$$
\begin{aligned}
& \operatorname{mes}\left\{t: T_{n} a(t)>\tau\right\} \leqslant \sum_{k=1}^{s} \sum_{U \in \mathscr{A}_{k}}\left(\sum_{V \in \mathscr{A}_{U}, S(V)>\tau} \operatorname{mes}\left\{t: T_{n} a(t)=S(V)\right\}\right. \\
&\left.+\sum_{V \in \mathscr{B}_{U}, S(V)>\tau} \operatorname{mes}\left\{t: T_{n} a(t)=S(V)\right\}\right) \\
& \leqslant \sum_{k=1}^{s} \sum_{U \in \mathscr{A}_{k}, S(U)>\tau / 2}\left(\frac{(n-2 k) !}{n !} \cdot \frac{(n-k) !}{k !(n-2 k) !}\right. \\
&\left.+\frac{(n-2 k+1) !}{n !} \cdot \frac{(n-k) !}{(k-1) !(n-2 k+1) !}\right) \\
& \leqslant 2 \sum_{k=1}^{s} \sum_{U \in \mathscr{A}_{k}, S(U)>\tau / 2} \frac{(n-k) !}{(k-1) ! n !} \cdot
\end{aligned}
$$

Оценим теперь снизу функцию распределения случайной величины $H_{m} a(t)$. Для каждого $U \in \mathscr{A}_{k}, S(U)>\tau / 2$, через $F_{U}$ обозначим множество всех $t \in$ $[0,1]$, для которых найдутся множество $W \subset\{1, \ldots, m\}$ и взаимно однозначное соответствие $\sigma: W \rightarrow U$, такие, что $|W|=k$ (считаем, что $m \geqslant n$ ) и $h_{m, j}(t)=$ $a_{\sigma(j)}$, если $j \in W$, и $h_{m, j}(t)=0$ для всех $j \notin W$. Тогда, прежде всего, для $t \in F_{U}$

$$
H_{m} a(t)=\sum_{j=1}^{m} h_{m, j}(t)=S(U)>\frac{\tau}{2}
$$

Кроме того, ввиду независимости функций $h_{m, j}(t)(j=1, \ldots, m)$

$$
\begin{aligned}
\operatorname{mes}\left(F_{U}\right) & =C_{m}^{k} k ! \frac{1}{(m n)^{k}}\left(1-\frac{1}{m}\right)^{m-k} \\
& =\frac{m(m-1) \cdots(m-k+1)}{m^{k}}\left(1-\frac{1}{m}\right)^{m-k} \frac{1}{n^{k}} .
\end{aligned}
$$

Tak кaK

$$
\lim _{m \rightarrow \infty} \frac{m(m-1) \cdots(m-k+1)}{m^{k}}=1 \quad \text { и } \quad \lim _{m \rightarrow \infty}\left(1-\frac{1}{m}\right)^{m-k}=\frac{1}{e}>\frac{1}{3},
$$

TO

$$
\operatorname{mes}\left(F_{U}\right)>\frac{1}{3} \cdot \frac{1}{n^{k}}
$$

для всех достаточно больших $m \in \mathbb{N}$ и всех $k \leqslant s$.

Заметим, что в случае, когда $U \neq U^{\prime}$, мы имеем $F_{U} \cap F_{U^{\prime}}=\varnothing$. Действительно, если $i \in U \backslash U^{\prime}$, то для любого $t \in F_{U}$ найдется такое $j \in\{1, \ldots, m\}$, что $h_{m, j}(t)=a_{i}$. В то же время если $t \in F_{U^{\prime}}$, то либо $h_{m, j}(t)=a_{l} \neq a_{i}$, либо 
$h_{m, j}(t)=0 \neq a_{i}$. Поэтому ввиду оценок (20) и (18), а также леммы 7 получаем

$$
\begin{aligned}
\operatorname{mes}\left\{t: 2 H_{m} a(t)>\tau\right\} & =\sum_{k=1}^{s} \sum_{U \in \mathscr{A}_{k}, S(U)>\tau / 2} \operatorname{mes}\left(F_{U}\right) \geqslant \frac{1}{3} \sum_{k=1}^{s} \sum_{U \in \mathscr{A}_{k}, S(U)>\tau / 2} \frac{1}{n^{k}} \\
& \geqslant \frac{1}{6} \sum_{k=1}^{s} \sum_{U \in \mathscr{A}_{k}, S(U)>\tau / 2} \frac{(n-k) !}{(k-1) ! n !} \geqslant \frac{1}{12} \operatorname{mes}\left\{t: T_{n} a(t)>\tau\right\},
\end{aligned}
$$

и лемма доказана, если $n \geqslant 4$.

Если же $1 \leqslant n<4$, то простая проверка (см. рассуждения перед соотношением (20)) показывает, что

$$
\operatorname{mes}\left\{t: T_{n} a(t)>\tau\right\} \leqslant 5 \operatorname{mes}\left\{t: 2 H_{m} a(t)>\tau\right\}
$$

для всех достаточно больших $m \in \mathbb{N}$ и $\tau>0$.

Замечание 9. В то же время оценка

$$
\operatorname{mes}\left\{t: T_{n} a(t)>\tau\right\} \leqslant C \operatorname{mes}\left\{t: H_{n} a(t)>\tau\right\} \quad(\tau>0)
$$

не верна ни при каком $C>0$, не зависящем от $n \in \mathbb{N}$. Действительно, если $a_{1}=\cdots=a_{n}=1$, то

a

$$
\operatorname{mes}\left\{t: T_{n} a(t)=n\right\}=\frac{1}{n !},
$$

$$
\operatorname{mes}\left\{t: H_{n} a(t)=n\right\}=\frac{1}{n^{n}} \text {. }
$$

\section{§5. Свойство Круглова и случайные перестановки}

Теорема 10. Пусть $E-$ r.i. пространство. Для того чтобы оператор $K$ был ограничен в $E$, необходимо и достаточно, чтобы последовательность операторов $T_{n}$ была равномерно ограничена в этом пространстве.

Доказательство. Мы будем использовать обозначения (3), (12) и (13).

Необходимость. По лемме 8 для произвольных $n \in \mathbb{N}, a=\left(a_{1}, \ldots, a_{n}\right) \geqslant 0$, $\tau>0$ и всех достаточно больших $m \in \mathbb{N}$

$$
\operatorname{mes}\left\{t: T_{n} a(t)>\tau\right\} \leqslant 12 \operatorname{mes}\left\{t: 2 H_{m} a(t)>\tau\right\} .
$$

Как уже отмечалось в начале предыдущей части, $H_{m} a \Rightarrow K f_{a}$ при $m \rightarrow \infty$. Следовательно [14, §6.2],

$$
\operatorname{mes}\left\{t: H_{m} a(t)>\tau\right\} \rightarrow \operatorname{mes}\left\{t: K f_{a}(t)>\tau\right\} \quad(m \rightarrow \infty)
$$

для всех $\tau>0$, в которых правая часть последнего соотношения непрерывна (т. е. всюду, кроме не более чем счетного множества). Поэтому из предыдущего неравенства следует, что для всех таких $\tau$

$$
\operatorname{mes}\left\{t: T_{n} a(t)>\tau\right\} \leqslant 12 \operatorname{mes}\left\{t: 2 K f_{a}(t)>\tau\right\} .
$$

Так как обе функции в этом неравенстве монотонны и непрерывны справа по $\tau$, то оно справедливо для всех $\tau>0$.

Хорошо известно $[10, \S 2.4 .3]$, что из неравенства

$$
\operatorname{mes}\{t:|x(t)|>\tau\} \leqslant C \operatorname{mes}\{t:|y(t)|>\tau\} \quad(\tau>0)
$$


и того, что $y \in E$, вытекает, что $x \in E$ и $\|x\|_{E} \leqslant \max (C, 1)\|y\|_{E}$ для любого r. i. пространства $E$. Таким образом, ввиду последнего соотношения

$$
\left\|T_{n} f_{a}\right\|_{E} \leqslant 24 \cdot\left\|K f_{a}\right\|_{E} \quad \text { или } \quad \sup \left\{\left\|T_{n} f_{a}\right\|_{E}:\left\|f_{a}\right\| \leqslant 1\right\} \leqslant 24 \cdot\|K\|_{E} .
$$

Из определения операторов $T_{n}$ следует, что $T_{n} x=T_{n} f_{a_{n}(x)}$, где $a_{n}(x)=$ $\left(a_{n, k}(x)\right)_{k=1}^{n}, a_{n, k}(x)=n \int_{(k-1) / n}^{k / n} x(s) d s$. Так как $\left\|f_{a_{n}(x)}\right\|_{E} \leqslant\|x\|_{E}[10, \S 2.3 .2]$, а пространство $E$ сепарабельно или совпадает со своим вторым двойственным, то в итоге

$$
\sup _{n}\left\|T_{n}\right\|_{E} \leqslant 24 \cdot\|K\|_{E}
$$

Достаточность. Предположим, что $\sup _{n}\left\|T_{n}\right\|_{E}=C<\infty$. В силу леммы 6 и снова $[10, \S 2.4 .3]$

$$
\left\|H_{m} f_{a}\right\|_{E} \leqslant 3\left\|T_{n m}\right\|_{E}\left\|f_{a}\right\|_{E} \leqslant 3 C\left\|f_{a}\right\|_{E}
$$

Так как $H_{m} f_{a} \Rightarrow K f_{a}$ при $m \rightarrow \infty$, то отсюда ввиду [1, предложение 1.5 ] получаем

$$
\left\|K f_{a}\right\|_{E^{\prime \prime}} \leqslant 3 C\left\|f_{a}\right\|_{E} .
$$

Пусть теперь функция $f=f^{*} \in E$ произвольна. Если

$$
f_{n}(t)=\sum_{k=1}^{2^{n}} f\left(k 2^{-n}\right) \chi_{\left((k-1) 2^{-n}, k 2^{-n}\right)}(t) \quad(0 \leqslant t \leqslant 1, n \in \mathbb{N}),
$$

то $f_{n}(t) \uparrow f(t)$ п. в. и, значит, $f_{n} \Rightarrow f[14, \S 6.2]$. Поэтому $\varphi_{n}(t) \rightarrow \varphi(t)(t \in \mathbb{R})$, где $\varphi_{n}(t)$ и $\varphi(t)$ - характеристические функции случайных величин $f_{n}$ и $f$ соответственно $[14, \S 6.4]$. Так как, согласно $[1,1.6], \varphi_{K \xi}(t)=\exp \left(\varphi_{\xi}(t)-1\right)$ для любой случайной величины $\xi$, то $\varphi_{K f_{n}}(t) \rightarrow \varphi_{K f}(t)(t \in \mathbb{R})$, т. е. $K f_{n} \Rightarrow K f$. Кроме того, ввиду (21)

$$
\left\|K f_{n}\right\|_{E^{\prime \prime}} \leqslant 3 C\left\|f_{n}\right\|_{E} \leqslant 3 C\|f\|_{E} \quad(n \in \mathbb{N}) .
$$

Поэтому, применяя еще раз [1, предложение 1.5$]$, получаем $\|K f\|_{E^{\prime \prime}} \leqslant 3 C\|f\|_{E}$. Так как функция распределения величины $K f$ зависит лишь от функции распределения величины $f$, то последнее означает, что $K$ - ограниченный оператор из $E$ в $E^{\prime \prime}$. Тем самым результат получен, если $E=E^{\prime \prime}$. Осталось рассмотреть случай, когда $E \neq E^{\prime \prime}$. Тогда пространство $E$ сепарабельно.

Прежде всего, используя тот факт, что любая функция $f \in E^{\prime \prime}, f \geqslant 0$, является пределом п. в. своих «срезок» $\tilde{f}_{n}:=f \chi_{\left\{f_{n} \leqslant n\right\}}(n \in \mathbb{N})$, и рассуждая точно так же, как и ранее, можно доказать ограниченность оператора $K$ в пространстве $E^{\prime \prime}$. Поэтому ввиду [3, теорема 7.2] функция

$$
g(t):=\frac{\ln (e / t)}{\ln (\ln (\ln (a / t)))},
$$

где $a>0$ достаточно велико, принадлежит $E^{\prime \prime}$. Таким образом, если

$$
\psi(u):=\frac{u \ln (e / u)}{\ln (\ln (\ln (a / u)))} \quad(0<u \leqslant 1),
$$


то $M_{\psi} \subset E^{\prime \prime}$. Отсюда следует, что $\left(M_{\psi}\right)_{0} \subset\left(E^{\prime \prime}\right)_{0}=E_{0}=E$ в силу сепарабельности пространства $E$. Легко проверить, что

$$
h(t):=\frac{\ln (e / t)}{\ln (\ln (a / t))} \in\left(M_{\psi}\right)_{0}
$$

и, значит, $h \in E$. Отсюда и из [3, теорема 4.4] следует, что

$$
K: L_{\infty} \rightarrow E .
$$

Пусть теперь $f \in E$. Ввиду сепарабельности пространства $E$ существует такая последовательность $\left\{f_{n}\right\} \subset L_{\infty}$, что $\left\|f_{n}-f\right\|_{E} \rightarrow 0$. Таким образом, из того, что $K: E \rightarrow E^{\prime \prime}$, следует, что $\left\|K f_{n}-K f\right\|_{E^{\prime \prime}} \rightarrow 0$. С другой стороны, в силу $(22)$ и изометричности вложения пространства $E$ в $E^{\prime \prime}$ получаем, что $\left\{K f_{n}\right\} \subset E$, а значит, $K f \in E$.

Замечание 11. Из доказательства теоремы вытекают следующие оценки для произвольного r. і. пространства $E$ :

$$
\frac{1}{24} \sup _{n}\left\|T_{n}\right\|_{E} \leqslant\|K\|_{E} \leqslant 3 \sup _{n}\left\|T_{n}\right\|_{E} .
$$

Приведем некоторые следствия, вытекающие из теоремы 10 . Пусть $n \in \mathbb{N}$, $S_{n}$ - множество перестановок чисел $1, \ldots, n$, а $l=l_{n}$ - произвольное взаимно однозначное отображение множества $S_{n}$ на множество $\{1, \ldots, n !\}$. Распространим оператор $A_{n}$, определенный ранее на $\mathbb{R}^{n}$ соотношением (3), на пространство матриц $x=\left(x_{i, j}\right)_{1 \leqslant i, j \leqslant n}$ следующим образом:

$$
A_{n} x(t)=\sum_{i=1}^{n} x_{i, \pi(i)}, \quad t \in\left(\frac{l(\pi)-1}{n !}, \frac{l(\pi)}{n !}\right) .
$$

Один из главных результатов работы [8] (см. следствие 8) состоит в том, что условие равномерной ограниченности операторов, порожденных случайными перестановками, на множестве диагональных матриц является достаточным для их равномерной ограниченности на множестве всех матриц. Применяя теорему 10, мы получаем

Следствие 12. Если $E \in \mathbb{K}$, то для любого $n \in \mathbb{N}$ и любой матрицъ $x=$ $\left(x_{i, j}\right)_{1 \leqslant i, j \leqslant n}$

$$
\left\|A_{n} x\right\|_{E} \leqslant C\left(\left\|\sum_{k=1}^{n} x_{k}^{*} \chi_{\left(\frac{k-1}{n}, \frac{k}{n}\right)}\right\|_{E}+\frac{1}{n} \sum_{k=n+1}^{n^{2}} x_{k}^{*}\right),
$$

где $\left(x_{k}^{*}\right)_{k=1}^{n^{2}}-$ перестановка последовательности $\left(\left|x_{i, j}\right|\right)_{i, j=1}^{n}$ в невозрастаящем порядке, а $C>0$ не зависит ни от $n$, ни от $x$.

Следствие 13. Для того чтобы операторы $T_{n}$ были равномерно ограничены в пространстве Орлича $\exp L_{p}$, необходимо и достаточно, чтобы $p \leqslant 1$.

Действительно, это утверждение вытекает из теоремы 10 и того, что условие $p \leqslant 1$ эквивалентно ограниченности оператора $K$ в пространстве $\exp L_{p}[1,2.4$, c. 42$]$.

Из теоремы 10 и следствия 3 вытекает 
Следствие 14. Для любого r. i. пространства $E$, maкого, что $\sup _{n}\left\|T_{n}\right\|_{E}<$ $\infty$, существует $r$. $i$. пространство $F \varsubsetneqq E$, для которого также $\sup _{n}\left\|T_{n}\right\|_{F}<$ $\infty$.

Для данного r. i. пространства $E$ и $p>1$ через $E(p)$ обозначим пространство всех измеримых на $[0,1]$ функций $x=x(t)$, таких, что $|x|^{p} \in E$, с нормой

$$
\|x\|_{E(p)}=\left\||x|^{p}\right\|_{E}^{1 / p} .
$$

Хорошо известно, что $E(p) \subset E$ и что $\|x\|_{E} \leqslant\|x\|_{E(p)}$ для всех $x \in E(p)[9,1 . \mathrm{d}]$. Пусть $E$ и $F-$ r.i. пространства, $E \subset F$ и оператор $K$ ограничен в $E$. Отсюда, вообще говоря, не вытекает ограниченность этого оператора в $F[3$, следствия 5.6 и 5.7]. Однако справедливо

Следствие 15. Если оператор $K$ ограничен в пространстве $E(p)$, то он ограничен и в $E$.

Доказательство. Ввиду теоремы 10 достаточно доказать, что из равномерной ограниченности операторов $T_{n}$ в $E(p)$ следует их равномерная ограниченность в $E$.

Пусть $x=\left(x_{1}, \ldots, x_{n}\right) \in \mathbb{R}^{n}, x \geqslant 0$ и $\left\|T_{n} x\right\|_{E(p)} \leqslant C\|x\|_{E(p)}(n \in \mathbb{N})$. Это означает, что

$$
\left\|\left(T_{n} x\right)^{p}\right\|_{E}^{1 / p} \leqslant C\left\|x^{p}\right\|_{E}^{1 / p}
$$

После замены $x^{p}=y$ получим

$$
\left\|\left(T_{n} y^{1 / p}\right)^{p}\right\|_{E} \leqslant C^{p}\|y\|_{E} .
$$

Так как из определения операторов $T_{n}$ следует, что $\left(T_{n} y^{1 / p}\right)^{p} \geqslant T_{n} y$, то, согласно предыдущему соотношению, $\left\|T_{n} y\right\|_{E} \leqslant C^{p}\|y\|_{E}$, т. е. $T_{n}$ равномерно ограничены в $E$.

\section{ЛитеРАТУРА}

[1] M. Sh. Braverman, Independent Random Variables and Rearrangement Invariant Spaces, London Math. Society Lecture Note Series, vol. 194, Cambridge Univ. Press, Cambridge, 1994.

[2] В. М. Круглов, Замечание о бесконечно делимых распределениях, Теория вероятн. и примен., 15 (1970), 331-336.

[3] S. V. Astashkin, F. A. Sukochev, Series of independent random variables in rearrangement invariant spaces: an operator approach, Israel J. Math., 145 (2005), 125-156.

[4] С. В. Асташкин, Ф. А. Сукочев, Сравнение сумм независимых и дизгюнктных функиий в симметричных пространствах, Матем. заметки, 76:4 (2004), 483-489.

[5] W. Johnson, G. Schechtman, Sums of independent random variables in rearrangement invariant function spaces, Ann. Probab., 17:2 (1989), 789-808.

[6] S. Kwapień, C. Schütt, Some combinatorial and probabilistic inequalities and their applications to Banach space theory, Stud. Math., 82:1 (1985), 91-106.

[7] Е. М. Семенов, Операторные свойства случайных перестановок, Функц. анализ и его прил., 28:3 (1994), 82-85.

[8] S. Montgomery-Smith, E. M. Semenov, Random rearrangements and operators, in: Amer. Math. Soc. Transl. Ser. 2, vol. 184, Amer. Math. Soc., Providence, RI, 1998, $157-183$.

[9] J. Lindenstrauss, L. Tzafriri, Classical Banach Spaces II. Function spaces, SpringerVerlag, Berlin-Heidelberg-New York, 1979. 
[10] С. Г. Крейн, Ю. И. Петунин, Е. М. Семенов, Интерполяиия линейных операторов, Наука, M., 1978.

[11] J. V. Ryff, Orbits of $L_{1}$-functions under doubly stochastic transformations, Trans. Amer. Math. Soc., 117 (1965), 92-100.

[12] G. G. Lorentz, Relations between function spaces, Proc. Amer. Math. Soc., 12 (1961), $127-132$.

[13] М. Холл, Комбинаторика, Мир, М., 1970.

[14] А. А. Боровков, Теория вероятностей, Наука, М., 1976.

Самарский госуниверситет

e-mail: astashkn@ssu.samara.ru

School of Computer Science, Engineering and Mathematics

Flinders University, Australia

e-mail: zani0005@infoeng.flinders.edu.au

Воронежский госуниверситет

e-mail: semenov@func.vsu.ru

School of Mathematics and Statistics

University of New South Wales, Kensington, Australia

e-mail: f.sukochev@unsw.edu.au
Поступило в редакцию 28 ноября 2007 г. 